\title{
La formación de profesionales desde la reflexión en la acción con elementos fundamentales de la pedagogía crítica
}

\author{
Sandra Acevedo Zapata ${ }^{1}$
}

\begin{abstract}
Resumen
Objetivo. Determinar y analizar los elementos epistemológicos fundamentales para dar pertinencia a la formación profesional.

Método. Estudio descriptivo de carácter cualitativo, que recoge el análisis documental de cuatro categorías: como epistemología de la pedagogía, didáctica, pedagógica crítica y formación profesional.
\end{abstract}

Resultado. Se encontró que la formación pertinente con las necesidades de la sociedad, implica un compromiso con procesos de investigación de los docentes protagonistas, centrados en el proceso de formación, analizando el diseño, la implementación y evaluación de la vivencia del currículo. Todo esto a la luz de los fundamentos epistemológicos de la pedagogía crítica.

Palabras clave: Formación profesional, pedagogía crítica, formación teórica y formación práctica. 


\title{
The formation of professionals from the reflection in action with key elements of critical pedagogy
}

\begin{abstract}
Objective. Identify and analyze the fundamental epistemological elements to give relevance to vocational training.

Method. Qualitative descriptive study, which includes the documentary analysis of four categories: epistemology of pedagogy, didactics, pedagogical criticism and training.

Result. It was found that the relevant training to the needs of society, implies a commitment to research processes of the protagonists teachers, focusing on the training process, analyzing the design, implementation and evaluation of the experience of the curriculum. All this in the light of the epistemological foundations of critical pedagogy.
\end{abstract}

Keywords: Professional formation, critical pedagogy, theoretical and practical formation.

\section{Pedagogía critica desde la teoría educativa crítica}

Vale la pena empezar señalando desde la pregunta ¿Cómo realizar procesos de formación pertinentes?, ha llevado a realizar un análisis documental sobre alternativas que superan la mirada instrumental y reduccionista en que se puede caer al desconocer estos elementos que aporta el saber pedagógico. Todo esto con el fin de encontrar elementos epistemológicos que brinden insumos para orientar las prácticas de diseño e implementación de currículos en la formación de profesionales, desde la perspectiva de la pedagogía crítica, para la formación de profesionales capaces de reflexionar sobre su acción. 
Así las cosas la fundamentación de la formación de profesionales dentro del enfoque crítico-social en lo que corresponde una pedagogía crítica en cuanto la pedagogía es la reflexión y desarrollo de un conjunto concreto de prácticas, las cuales llevan a determinadas formas sociales, conocimientos, experiencias y subjetividades específicas.

En este sentido, una pedagogía crítica es el esfuerzo que desde los actos educativos se realiza para desvelar las ideologías dominantes y proponer contraideologías en aras de sociedades más democráticas "tenemos que activar las iniciativas ciudadanas, la vida municipal, las soluciones locales a problemas locales, todo ello dentro de un marco formal de división de poderes, elecciones transparentes y fiscalización de las autoridades. Eduquemos a los latinoamericanos para ejercer el poder. No el poder sobre los demás, sino el poder con los demás" (Fuentes, 1998: 23).

Esta postura pedagógica debe cruzar necesariamente por el convencimiento de los profesores sobre la acción que ejercen y el papel social que juegan. En otras palabras, No basta con decir nos toca adaptarnos a una sociedad en constante cambio, compleja, caótica sino que es necesario construir los referentes para que esos dichos y prácticas se concreticen y tanto personas como instituciones puedan desarrollar su subjetividad y su objeto social.

\section{Características de la pedagogía crítica}
a. La pedagogía es vista como hecho social;
b. La pedagogía es vista como hecho político y cultural;
c. La pedagogía crítica ayuda a construir problematizaciones que lleven a nuevas búsquedas.

\section{a. La pedagogía es vista como hecho social}

La acción pedagógica está inmersa en sociedades y culturas específicas y por lo tanto representa expectativas e intereses concretos de una época histórica, por lo tanto, es necesario, desarrollar un lenguaje que permita develar y comprender el nexo existente entre educación escolar, relaciones sociales y necesidadescompetencias que los estudiantes llevan a la escuela con el fin que se conviertan en punto de partida de los procesos educativos y escolares. En fin, configurar los aprendizajes en una perspectiva social.

Implicaciones curriculares desde la pedagogía como hecho social significa establecer los procedimientos para construir un proyecto cultural.

- El currículo es una forma de acceder al conocimiento, pero ese acceso no se hace de cualquier manera. 
-La teoría y el diseño del currículo tiene un compromiso con los entrecruzamientos de los elementos que hacen parte de la realidad social. -El currículo tiene como finalidad un proceso de cambio y transformación de la realidad.

-En el diseño curricular se debe tomar como punto de partida los problemas reales y concretos con que hoy se enfrentan profesores, estudiantes y comunidad educativa en general.

-El currículo debe operar bajo la convicción que las escuelas, son lugares de lucha y de posibilidad.

\section{b. La pedagogía es vista como hecho político y cultural}

Si al decir de muchos, las instituciones educativas son el mejor espacio para vivir la democracia, es innegable que esas vivencias hay que construirlas y sobretodo la institución debe organizar su funcionamiento interno, de tal forma que ella misma sea ejemplo de tal situación.

Se trata de construir la actitud política, no ya desde los referentes de otros tiempos, en los cuales se ligaba al partido, y este le definía a sus seguidores qué hacer, qué no hacer y qué deshacer. Ahora lo político tiene que ver con la participación, con la toma de decisiones producto de la discusión y de la argumentación.

Implicaciones curriculares desde la pedagogía como hecho político y cultural

-El cuestionamiento del sujeto y de las instituciones de las posiciones y las actitudes teóricas y prácticas que asumen.

-Tener en cuenta que la ciencia social crítica, combina la reestructuración de lo que se conoce como crítica, con la voluntad política de actuar.

\section{c. La pedagogía crítica ayuda a construir problematizaciones que lleven a nuevas búsquedas}

En este sentido, una pedagogía crítica parte de la base que la realidad social en sus diferentes órdenes no es estática y que por lo tanto, de lo que se trata es de una lucha colectiva sobre el mundo material, utilizando para ello la imaginación social, dicho en otras palabras, la pedagogía crítica se dirige a un proceso de interpenetración de horizontes, de fusión de horizontes de sentido.

Ayudar a que se mantenga viva la capacidad de las nuevas generaciones para razonar, preguntar y criticar mientras, entre todos, reconstruimos los programas para una nueva esperanza y evitamos que, con la excusa del fin de la historia, lo que paren de verdad sean nuestras posibilidades de cambiar el presente y construir un futuro mejor (Fontana, 1992:30). 
Desde esta perspectiva la pedagogía en general y la pedagogía crítica en particular, como disciplina buscan constituir su objeto y método de estudio, así como los conceptos que la atraviesan como tal, en palabras de Díaz: "en este contexto, han mediado un tanto intereses por: a) establecer la legitimidad epistemológica de este campo de estudio al intentar definir su estatuto paradigmático de disciplina, b) definir su carácter mediático en función de la transmisión social y cultural de órdenes simbólicos dominantes o, c) favorecer los problemas especificos de su heterogeneidad contextual tales como procesos comunicativos, actores, situaciones relaciones sociales, modelos pedagógicos, innovaciones, etc." (Díaz, 1990: 41).

En Colombia, los investigadores más representativos de este enfoque del grupo de historia de las practicas pedagógicas, donde la pedagogía se concibe como saber y como practica no se agota ni se explica en un único paradigma o enfoque, por el contrario, de acuerdo con el pedagogo alemán Chistoph Wulf(1991), quien afirma que los diferentes paradigmas que podrían conformar una epistemología de las ciencias de la educación, poseen su respectivo campo de legitimidad que logra relativizarse por fuerza del pluralismo que caracteriza actualmente la producción conceptual en la ciencia humanas y sociales.

Los planteamientos anteriores nos permiten identificar una característica común, que es compartida por los diferentes enfoques pedagógicos antes enunciados, esta es la "la postura crítica acerca de la educación tradicional, que privilegiaba las concepciones y los métodos autoritarios de la enseñanza, el aprendizaje que atomizaban el conocimiento y asumían al estudiante y al docente como objetos pasivos y aislados del contexto histórico social y cultural".

La diferencia de las actuales concepciones pedagógicas, en lo fundamental radica, en la intención de privilegiar el enfoque o la mirada que ha de orientar el análisis y la interpretación de la forma como se aprende y se interactúa con el conocimiento, el lenguaje, el trabajo y la sociedad. En este sentido se destacan, las distintas posturas que sobre los diversos roles pueden asumir los maestros, los estudiantes, la comunidad, la institución educativa y el sector productivo, en relación con el conocimiento y su aplicación en beneficio del bien común.

Dentro de este panorama pedagógico, es notable la importancia que en las últimas décadas, han presentado las distintas concepciones de origen constructivista, que van desde las puramente cognitivas (construcción de mapas conceptuales a finales de la década del 70), pasando por las que enfatizaban la epistemología de las disciplinas, hasta llegar al nuevo constructivismo, sustentado en cuatro concepciones: la teoría crítica de Habermas (1982); la fenomenología de mundo de la cotidianidad de Husserl, Schütz (1984); las relaciones de poder y su incidencia en el discurso y la práctica educativa, y en el acceso y la producción del conocimiento de Bernstein (1975) y los desarrollos del aprendizaje comprensivo de Gardner (2000). 
En correspondencia con los principios que orientan el proyecto educativo de las instituciones de educación superior, y de acuerdo con la naturaleza de los programas profesionales, cuya esencia reside en la creatividad y la aplicación del conocimiento científico, tecnológico y social, las nuevas concepciones del constructivismo adquieren sentido y significado pedagógico para lograr los propósitos y objetivos misionales y formativos de la institución y los programas. En este sentido, la pedagogía es un instrumento de reflexión y formación integral para la adquisición de valores, competencias y habilidades cognoscitivas.

Consecuente con esta concepción pedagógica, la universidad, asume la didáctica como un proceso que se aborda desde la perspectiva investigativa como objeto de la reflexión pedagógica que permite innovar el desarrollo de las metodologías y los medios más adecuados que faciliten el encuentro estimulante de estudiantes y profesores para el desarrollo de la inventiva, la construcción y la re-creación de conocimiento, y transforme los espacios educativos en escenarios de análisis y estudios de los problemas de interés, pertinentes a los programas académicos y relacionados con las problemáticas del país.

Para concretar esta concepción de didáctica en los procesos formativos, se requiere profesores cualificados, que interioricen los métodos conducentes a descubrir las significaciones epistemológicas de los propios fenómenos y de la ciencia, con espíritu crítico y analítico.

\section{Teoría critica educativa}

La teoría crítica propone una interpretación de la sociedad a través de la razón emancipadora que conduzca a los seres humanos a analizar las estructuras de la sociedad y las formas sociales que la sociedad capitalista ha establecido, para por ese camino construir una sociedad buena, humana y racional (Mardones, 1991: 38). Así las cosas, Adorno va a plantear que las ciencias sociales deben ocuparse de los problemas reales en donde se hallan las verdaderas contradicciones sociales. Alfred Schütz (1984), afirma que:

El objetivo primario de las ciencias sociales es lograr un conocimiento organizado de la realidad social: si ese conocimiento es organizado tiene que hacer referencia su carácter objetivo, pero al igual al subjetivo. Al igual plantea que la realidad social contiene elementos de creencias y convicciones que son reales porque así lo definen los participantes y por lo tanto se trata de dilucidarlas y debatirlas. Se pregunta ¿cómo es posible elaborar conceptos objetivos y una teoría objetivamente verificable de las estructuras subjetivas de sentido? 
Es en este sentido que la teoría crítica adopta el enfoque crítico-social el cual tiene varias características que lo conforman como tal: dialéctico; emancipatorio; que analiza la vida cotidiana, las relaciones de poder, la acción social colectiva, las estructuras de clases y las ideologías que las sustentan; de igual forma, las potencialidades comunitarias. En síntesis, el enfoque crítico-social es valorativo, popular, construido colectivamente y en él el sujeto toma parte de manera protagónica, comprometido políticamente en aras de la transformación social la cual tiene como referente fundamental la libertad y la justicia social. De esta manera, estamos de acuerdo con Luhmann (1984), para el cual la realidad social ofrece un horizonte de gran amplitud de horizontes a la cual él llama complejidad, caracterizándose porque el entorno resulta complejo para el sistema.

Para ese análisis y propósito emancipador es necesario perfilar el tipo de sociedad que se busca, de tal forma que el mismo dé sentido a la búsqueda que realizan las personas que las habitan, sentido que es parte fundamental de su individualidad y que puede conducir al camino de la subjetividad, son los seres humanos quienes le dan sentido a sus vidas y a las sociedades en las cuales interactúan y realizan sus acciones; por lo tanto, parte de un postulado que aunque no rechaza la objetividad, sí la coloca en un plano diferente, oponiéndose así a aquel postulado sociológico enunciado por Durkheim a comienzos del siglo XX y seguido por otras corrientes, de tratar los hechos sociales como cosas, "resulta más fácil tratar los hechos sociales como cosas o como personas que como relaciones" Bourdieu (1984: 69); sin embargo, los análisis de la sociedad no tienen como principio la facilidad, sino las relaciones, las interdependencias, la complejidad entre otros.

El enfoque crítico-social busca que las disciplinas científico-sociales se comprometan en los entrecruzamientos de los diferentes elementos que hacen parte de la realidad social, partiendo del hecho que es importante manejar saberes pero que éstos deben estar en estrecha relación con los diferentes aspectos de la realidad social como lo político, social, económico y cultural. En este sentido, el enfoque crítico-social se dirige a establecer "la conexión del saber teórico con una práctica vivida" (Habermas, 1982:384), lo cual le permite al sujeto establecer sus condiciones de posibilidad en como articulación de sus teorías y prácticas sobre la realidad social y su papel en las mismas.

El proceso de cambio y transformación de la realidad, la concepción de la conciencia crítica del hombre, el ser humano visto como ser histórico y estable, puntos característicos del enfoque crítico-social, es investigativo, integral y es multicultural; para lograr ello es necesaria la educación, combatiendo problemáticas sociales afincadas de la realidad: jerarquías clasistas altamente diferenciadas e inequitativas e injusticias marcadas. Sin negar que ha habido avances significativos en las últimas décadas en cobertura educativa y protección social como producto de la democratización institucional, aún se está lejos de 
satisfacer las demandas del conjunto de la población ¿Será que en épocas de recesión, crisis fiscal, endeudamiento externo, devaluación, neoliberalismo, apertura... los gobiernos lo van a hacer por su propia voluntad? Seguramente no; sólo en la medida que exista presión social acompañada de voluntad e iniciativa de la población se va a conseguir este propósito, lo cual nos obliga a releer el panorama social de tal forma que no reproduzcamos lo mismo y se contrarresten las prácticas dominadoras e ideologizadas y así:

"Ayudar a que se mantenga viva la capacidad de las nuevas generaciones para razonar, preguntar y criticar mientras, entre todos, reconstruimos los programas para una nueva esperanza y evitamos que, con la excusa del fin de la historia, lo que paren de verdad sean nuestras posibilidades de cambiar el presente y construir un futuro mejor" (Fontana 1993: 47).

La teoría crítica es tomada como referente en el campo educativo por los teóricos educativos críticos -liderados por Henry Giroux, Michael Apple, Paulo Freire entre otros- quienes reactivaron la discusión iniciada por Gramsci sobre los intelectuales a los que este último llamó orgánicos y los primeros llaman transformativos pero que en esencia se dirigen hacia lo mismo: la transformación de la sociedad.

Esa transformación en concepto de ambos debe direccionarse desde posturas críticas lo cual conduzca a la transformación de la realidad social. La postura crítica en concepto de Habermas tiene dos postulados básicos como lo son desvelar y transformar (Vasco: 1990), pero ¿Qué se desvela y qué se transforma? en concepto de todos los críticos sociales, las estructuras opresoras de la sociedad (Mardones: 1991).

Hoy día a las estructuras sociales se le ha sumado un aspecto fundamental, como lo es la constitución de la subjetividad; ello se hace más evidente si se considera que las ciencias sociales no tienen hoy día sólo compromiso con la realidad social como eje abstracto de las problemáticas que acontecen -o que sucedensino también con los individuos; es decir, que las disciplinas no construyen sus objetos y sus métodos sólo con el ánimo de abordar unos saberes sino con el objetivo de interactuar con las personas y la sociedades a las cuales van dirigidos esos saberes.

\section{El socio - constructivismo como modelo pedagógico de las universidades}

Se parte de asumir el acto pedagógico como la interacción dinámica entre los sujetos que participan en los procesos educativos que se desarrollan dentro y fuera del contexto de la escuela. En este sentido, los estudiantes y los docentes son agentes dinamizadores, creadores y socializadores del conocimiento, la 
tecnología, la cultura y los valores que identifican una sociedad en una época histórica. Por ello no existen personas carentes o vacías de conocimientos, sino llenas de conocimientos aparentes, que a la luz del pensamiento de Sócrates se encuentran "indigestas de falsos conocimientos", los cuales distorsionan no solo los conocimientos verdaderos, sino también la realidad.

La verdadera educación para este filósofo estaba centrada en el método y la orientación para conocer la verdad, para que cada persona fuera consciente de sus limitaciones y falsos conocimientos (conocimiento aparente), y así pudiera abrir su espíritu y su intelecto a los nuevos conocimientos, verdades y realidades, que habían logrado demostrar las inconsistencias y limitaciones de lo aparente. En todo ello lo que se resalta, es que la persona que aprende, es la protagonista de su propio aprendizaje y por consiguiente de su propio conocimiento. Igualmente, no existen personas carentes de experiencias y conocimientos, que se puedan asumir como receptores pasivos, mientras que otros podrían asumirse como los únicos poseedores del conocimiento y la verdad.

Estos fundamentos gnoseológicos y pedagógicos son los que han orientado las escuelas de pensamiento educativo que se han considerado activas, como la Escuela Nueva de Montessori y Decroly, predecesoras del constructivismo pedagógico, que concibe el verdadero aprendizaje humano, como una construcción de cada alumno que logra modificar su estructura mental y alcanzar un mayor nivel de diversidad, complejidad e integración; es decir, el verdadero aprendizaje es aquel que contribuye al desarrollo de la persona.

La enseñanza constructivista considera que el aprendizaje humano es siempre una construcción interior, aún en el caso de que el educador acuda a una exposición magistral, pues ésta no puede ser significativa si sus conceptos no encajan, ni se ensartan en los conceptos previos de los estudiantes, planteamiento que se halla ampliamente desarrollado en el capítulo sobre constructivismo y enseñanza por procesos, (Flórez, 1997).

Según Gardner, en la historia educativa de occidente se han identificado cuatro grandes objetivos: trasmitir roles y funciones; trasmitir valores culturales; inculcar una formación básica y comunicar determinados contenidos y maneras de pensar de carácter disciplinario, los cuales han estado enmarcados en tres grandes virtudes que han animado la educación, a saber: buscar dentro de la propia cultura, lo que es verdadero, lo que es bello y lo que es bueno. A pesar de estos ideales, las instituciones educativas han llevado a cabo estas actividades de distinta forma y en muchas ocasiones con concepciones contradictorias, como las siguientes:

- Entre la amplitud y la profundidad

- Entre la acumulación y la construcción de conocimientos

- Entre la obtención de unos resultados utilitarios y el desarrollo intelectual en sí 
- Entre la enseñanza uniforme y la enseñanza individualizada

- Entre una educación a cargo de grupos privados y la educación como responsabilidad pública

- Entre una educación que ignora o fusiona las disciplinas y una educación que destaca el dominio de las mismas

- Entre una educación que minimíza o crítica la evaluación y otra

- Entre unos niveles de exigencia relativos y matizados y unos niveles elevados y universales

- Entre una educación que favorece la tecnología y una educación que destaca la dimensión humana.

Ante cada de una de estas contradicciones, Gardner (2000), toma partido indicando: prefiero la profundidad a la amplitud; la construcción a la acumulación; la búsqueda del conocimiento en sí, al sometimiento a la utilidad; la enseñanza individualizada a la uniforme, y una educación de carácter público. Prefiero la educación centrada en el estudiante, a la centrada en el enseñante, y apoyo a la que presta atención a las diferencias individuales y de desarrollo. Gardner (2000), también indica, que es partidario de la educación, firmemente enraizada en las disciplinas, de emplear la evaluación con regularidad y de establecer unos niveles de exigencia elevados y universales, por último indica, que las nuevas tecnologías son extraordinariamente prometedoras, pero se deben ver como medios y no como un fin.

En esencia las principales características del constructivismo son las siguientes:

- Parte de reconocer la estructura conceptual de cada alumno, como los preconceptos y opiniones que cada estudiante tiene de los temas que se tratan en las diferentes clases.

- Orienta el cambio conceptual que se espera de la construcción activa del nuevo concepto, y su repercusión en la nueva estructura mental.

- Confronta las ideas y preconceptos afines al tema de interés, con el nuevo concepto científico que se intenta problematizar.

- Aplica el nuevo concepto a situaciones concretas relacionándolo con otros conceptos de diferentes disciplinas

\section{La necesidad de la formación de profesionales críticos y reflexivos}

Desde la perspectiva de la epistemología de la práctica, entendida como una reflexión en la acción, es decir: pensar en lo que se hace, mientras se está haciendo; se comprende que la práctica profesional ya sea del arquitecto, ingeniero, administrador o mercadotecnista, etc., no se agota con la aplicación técnica de los conocimientos adquiridos en la universidad. 
A su vez, estos conocimientos tampoco son suficientes cualitativamente, para comprender y transformar la realidad y sus problemáticas. Se propone entonces, una mirada integral que rompa el esquema de la racionalidad unidimensional de Martin Barbero (2002), y de cabida a la lógica de lo estético, lo sensible, lo lúdico y lo bello, que articulado con la racionalidad técnica, se constituya en la base que posibilite la "creatividad profesional".

Se hace referencia, cuando se menciona el carácter reflexivo de la acción práctica de los profesionales, tal como lo afirma Donald A, Schön (1992), al expresar que los "Centros de formación de profesionales en el marco de la estructura actual de la investigación universitaria otorgan un estatus privilegiado al conocimiento sistemático preferiblemente de carácter científico. La racionalidad técnica, epistemología de la práctica que más abunda en este tipo de centros, considera la competencia profesional, como la "aplicación del conocimiento privilegiado a los problemas instrumentales de la práctica" Schön (1992: 56).

La segmentación del conocimiento que caracteriza a los currículos normativos, no solamente obstaculiza la articulación de los saberes, que se enseñan y aprenden, sino que además distorsiona la labor investigativa de los docentes y estudiantes, lo cual minimiza la posibilidad de reflexionar sobre la práctica profesional, pues esta se reduce a un "hacer técnico", que se agota en la inmediatez, y no permite que el profesional analice y comprenda la acción y las múltiples relaciones que se generan con el entorno.

En esta perspectiva, se crea un nuevo rol para el docente universitario, el cual consiste en combinar la enseñanza de la ciencia aplicada, con la formación tutorizada o guiada, para crear la disposición reflexiva de la acción práctica, que permita desarrollar las competencias profesionales del "aprender a aprender y del aprender haciendo". Esta forma de reflexión, se constituye en la acción central, de la práctica profesional, que estimula la creatividad, para enfrentar y resolver situaciones de incertidumbre, singularidad y conflicto.

En síntesis, los profesionales formados con esta concepción desarrollan las capacidades para experimentar sorpresa y asombro, que los lleva a replantear sus conocimientos, sus posibles aplicaciones y resultados, más allá de las reglas, los hechos, las teorías y las operaciones disponibles. Este profesional así formado, está en capacidad de reaccionar ante lo inesperado o lo extraño, para lo cual puede a partir de su inventiva y creatividad, reestructurar algunas estrategias de acción, teorías de los fenómenos o modos de configurar un problema y crear nuevos experimentos y modelos sobre la marcha, para poner a prueba su comprensión. Estos profesionales, se comportan entonces, más como un investigador que trata de modelar un sistema experto, que como un "experto", cuyo comportamiento es modelado. 
Subyace en esta visión reflexiva de la acción, una concepción constructivista de la realidad, que concibe al profesional como un sujeto capaz de configurar sus mundos a partir de los conocimientos, percepciones, apreciaciones y creencias generadas en las diferentes vivencias, en aquello que Goodman (1968), denominó como la construcción del mundo.

A través de diferentes actos de codificación y descodificación, el ejercicio permanente de reconstrucción, clarificación y del establecimiento de sus propios límites, de acuerdo con sus capacidades y las reglas establecidas por las profesiones y la sociedad.

Los profesionales reflexivos, construyen una forma particular y profesional de ver el mundo y una forma de configurar y mantener el mundo según su visión. Cuando este profesional enfrenta la incertidumbre o algo inesperado, reconstruye su mundo, y con ello comprende y revela el proceso o la situación no clara, ni comprensible, que subyace en su práctica profesional. De esta manera se concreta que el "aprender a hacer y el aprender haciendo", constituyen un fundamento metodológico coherente con la concepción constructivista del aprendizaje que orienta esta propuesta curricular.

Se propone entonces una epistemología de la práctica que, con base en el "conocimiento de la acción y la reflexión de la acción", permita superar la visión tradicional de currículo normativo, que polariza la investigación y la práctica y no posibilita la formación de profesionales reflexivos con capacidad para enfrentar la incertidumbre, el conflicto, el caos y la singularidad de la vida real.

Tres consideraciones se plantean en este contexto para orientar la discusión y la formulación de nuevos argumentos en la formación de profesionales reflexivos, prácticos y pertinentes con su entorno, con capacidad para construir, reconstruir y mejorar dicho entorno:

* Los currículos de formación profesional no consultan la situación de los profesionales, en sus contextos de trabajo, que en muchos casos limitan la creatividad y por tanto su capacidad reflexiva.

* La necesidad urgente de desarrollar nuevas conexiones entra la ciencia aplicada y la reflexión en la acción.

* La necesidad de crear o revitalizar una epistemología de la práctica, que incluya como componente central la reflexión en la acción, que llevan a cabo los profesionales en la práctica en sus ámbitos laborales.

Estos requerimientos pueden satisfacerse mejor concediendo un lugar preponderante a la práctica reflexiva, como un contexto apropiado para la construcción de enlaces entre los escenarios laborales y los mundos de la 
universidad y de la práctica, y donde también el rol del docente como tutor adquiere significado e importancia relevante en los procesos enseñanza y aprendizaje (Shön, 1992).

\section{Características y fundamentos de la concepción del currículo}

En la perspectiva de Stenhouse (1984), el currículo es un proceso educativo, un curso de acción que desarrolla el profesor con sus estudiantes, una pauta viva de ordenamiento de la práctica de la enseñanza, un objeto simbólico y significativo de acción para maestros y alumnos que se encarna en palabras, imágenes sonidos, juegos y simulaciones; una secuencia de procedimientos hipotéticos que solo se puede comprender y confirmarse en la sesión concreta de la enseñanza.

En este sentido, el currículo siempre es hipotético porque cada vez necesita comprobarse su viabilidad y efectividad, en cuanto al desarrollo de procesos de construcción del conocimiento, que por su naturaleza se manifiestan de manera diversa y compleja de acuerdo con los distintos intereses y estilos de aprendizaje de los estudiantes y profesores, todo lo cual rompe con la concepción de pensamiento, metodologías y didácticas de corte dogmático y determinísticas (Flórez, 1984).

En consecuencia, cada tema, contenido y concepto desarrollado en un currículo, debe asumirse no como un resultado, sino de manera dinámica como un proceso, como interrogante constitutivo del mismo conocimiento, con miras a los propósitos más altos de la formación de la persona, mediante la construcción reflexiva de la ciencia y el arte, cuyos procesos y criterios no son ajenos a la formación del pensamiento propio y autónomo que se busca de dentro de la especialización en pedagogía y docencia universitaria.

Esta concepción de currículo por procesos asume la flexibilidad como uno de sus principios distintivos y esenciales, lo cual le permite estar en una permanente dinámica y disposición para el cambio, la influencia socio histórica y cultural de la comunidad y del país, en el cual se inscribe el programa de especialización, hasta el punto de que es la comunidad de la cual hacen parte los alumnos y profesores, la que a partir de la conciencia de sus propios problemas de supervivencia, de convivencia y proyección de futuro, dan pauta sobre los ejes temáticos principales que tendrán que formularse en el diseño curricular, bajo la forma de preguntas que asumirían y procesarían los estudiantes y profesores, no solo desde el saber universal de las ciencias, sino también desde el saber local vivo y activo de la tradición cultural de la misma comunidad.

Complementa esta concepción de currículo, los conceptos y criterios formulados por Fanny Forero (1999), para el diseño y estructuración de currículos universitarios en los cuales se destacan los siguientes aspectos: 
* Fundamentación: Una propuesta educativa debe basarse en alguno modelo o concepción de lo que se considera qué es la educación, los procesos de formación, la función social del conocimiento, los principios sobre el desarrollo humano y el aprendizaje. Además se debe fundamentar en la filosofía, los valores, la misión y la visión de las instituciones.

* Estructura: Es la forma como se deben seleccionar y organizar todas las intenciones, actividades, contenidos, metodologías, recursos y formas de coordinación y seguimiento. Es el armazón o el esqueleto sobre el que se construye el proceso educativo en las instituciones. Todo se estructura de acuerdo con unos principios y criterios previamente acordados y establecidos en el proyecto educativo institucional.

* Características: Son las condiciones que sirven de base para orientar la estructura del currículo, su desarrollo y evaluación. Estas características atraviesan toda la planeación, desarrollo y evaluación del currículo, entre ellas se destacan las siguientes:

- Pertinencia: Es la capacidad para responder a las necesidades y problemáticas del entorno; en este caso se relacionan con la necesidad de mejorar la calidad de los programas académicos a través de la cualificación de los profesores y por esta vía incidir en el impacto social de los proyectos, servicios educativos y egresados.

- Flexibilidad: Entendida en tres dimensiones que permiten al currículo una visión dinámica: Una primera, relacionada con la disposición para asumir el cambio en cuanto a los contenidos temáticos y problemáticos de los saberes y el saber hacer científico, técnico y tecnológico. Una segunda, entendida como la capacidad curricular para configurar diversas alternativas y ofertas de programas, contenidos que permitan a los estudiantes construir sus propios recorridos a partir de sus intereses y necesidades. Una tercera, referida a la capacidad de los programas para asumir los procesos de autoevaluación y sus resultados en términos de mejoramiento continuo.

Los créditos académicos se constituyen en una herramienta académica y administrativa que facilita y complementa la flexibilización curricular.

- Interdisciplinariedad: Desde el punto de vista curricular, se asume la interdisciplinariedad como una forma particular de organizar, desarrollar y comprender los diferentes proyectos, áreas temáticas o problemas que constituyen un plan de estudios, con la concurrencia sucesiva y articulada de saberes y disciplinas.

Un currículo concebido de manera interdisciplinaria, es un currículo estructurado y al mismo tiempo integrado por las diferentes visiones 
disciplinares, las cuales permiten abordar un problema o la realidad, desde diferentes ópticas y por tanto pensar en su solución y comprensión de una forma holística.

La interdisciplinariedad se manifiesta en la capacidad de los docentes, investigadores y estudiantes para trabajar en equipo y aportar conocimiento cualificado desde diferentes concepciones, con el propósito de contribuir en la búsqueda de alternativas de los diferentes problemas que convocan a la comunidad académica

- Universalidad: El conocimiento es una construcción universal y social a través de la cual se intenta explicar y comprender la realidad, los fenómenos naturales y los hechos que originan los distintos grupos humanos y sociales, lo cual hace que el conocimiento tenga una dimensión universal que lo valida intersubjetivamente, es decir su validez no está condicionada al contexto geográfico de su producción.

Por consiguiente, al institucionalizarse un saber en un currículo no pierde su exigencia de universalidad, por el contrario nutre y consolida el trabajo académico y curricular, y hace posible la libre circulación de conocimientos y la libertad de cátedra, enseñanza e investigación. La universalidad, también se refiere a la multiplicidad y extensión de ambientes y proyecciones que se derivan de un currículo y su sentido de ofrecer diferentes servicios educativos en escenarios geográficos diversos de su zona de influencia.

- Coherencia: Es el grado de correspondencia entre todos los componentes del currículo, y entre estos, con la filosofía, las políticas y el proyecto educativo de la institución. También se refiere al grado de correspondencia que debe existir entre los diferentes saberes, conocimientos y experiencias requeridos para formar un profesional universitario, o en cualquier otro nivel educativo de la educación superior.

- Eficiencia: Un currículo eficiente es aquel que está diseñado para alcanzar unos logros y productos académicos, en términos de profesionales formados con capacidad reflexiva y propositiva para comprender la realidad y resolver sus problemas de manera positiva; desarrollar investigación; transformar y aportar al desarrollo local y regional.

Un currículo también es eficiente cuando dispone, prevé y usa de manera adecuada y racional, los medios y recursos para el logro efectivo de sus objetivos.

En el Proyecto Educativo Institucional de las universidades que forman carreras relacionadas con el diseño y la producción artística, se asume el currículo como 
la expresión integral de los elementos esenciales que identifican los valores institucionales, éticos y democráticos que permiten formar profesionales con calidad humana, con capacidad para apropiar y aplicar la ciencia y la tecnología con responsabilidad y sentido critico

Hoy en día las universidades buscan lograr como características principales del currículo la flexibilidad, la pertinencia, la interdisciplinariedad y el compromiso social con la formación de profesionales con capacidad para promover el desarrollo local y regional y contribuir a la solución de sus principales problemáticas.

\section{La necesidad de una concepción de pedagogía}

En el mundo contemporáneo se identifican diferentes perspectivas y enfoques de la pedagogía que derivan de las distintas concepciones clásicas del pensamiento educativo, las cuales se nutren de las diversas corrientes paradigmáticas de las ciencias, la tecnología y la técnica que han predominado según los intereses prevalecientes en cada de las sociedades.

En Colombia, se han identificado en los últimos años diferentes enfoques pedagógicos en la orientación de la educación superior, entre los cuales, se destacan de manera particular los siguientes:

* Desde la perspectiva hermenéutica: la cual privilegia los procesos de reconstrucción del contexto cultural de la practica educativa, en el marco de los fines de la acción educativa, partiendo del supuesto que el saber pedagógico se crea sobre las experiencias cotidianas e inconscientes de los profesores, las cuales pueden ser objeto de reflexión y sistematización que conducen a la elaboración de pensamiento pedagógico.

* Desde la perspectiva integral y sistémica: centrada en la "Relación entre el discurso, la práctica y el espacio, considerados como elementos constitutivos del contexto de producción del discurso educativo, en el que están integrados las condiciones políticas, sociales, institucionales, técnicas y teóricas de reproducción-transformación de las relaciones sociales relevantes al campo de producción y a las modalidades de control simbólico".

En este enfoque, la pedagogía es asumida como un medio para la distribución del conocimiento, el poder y las formas de control, lo cual se realiza a través del currículo, las formas de transmisión del conocimiento y la evaluación. Esta perspectiva se sustenta en planteamientos de Basil Bernstein (1975) y sus seguidores en Colombia, entre los cuales se destacan los trabajos e investigaciones de los profesores Mario Díaz y Antanas Mockus. 
* Desde la perspectiva de una disciplina reconstructiva: este enfoque pedagógico se apoya en la teoría critica de Habermas, el cual parte de la posibilidad de reconstruir saber teórico a partir de un saber empírico e intuitivo que se manifiesta en las prácticas educativas relacionadas con los paradigmas y la enseñanza propios de cada las disciplinas y profesiones.

Esta perspectiva también se apoya en los postulados de Basil Bernstein (1975) y en las propuestas de la enseñanza para la comprensión de Howard Gardner y su grupo de investigación de Harvard.

* Desde la perspectiva de reconceptualización histórica de la pedagogía: centrada en los conocimientos producidos durante el desarrollo histórico de la pedagogía que continúan vigentes, en los conceptos mayores de los paradigmas educativos actuales (Ciencias de la educación, currículo y pedagogía), e igualmente en los conocimientos que otras disciplinas han construido sobre el campo conceptual de la pedagogía y la enseñanza, tales como formación, instrucción, aprendizaje, educación, escuela, aula y la didáctica entre otros.

Todo lo anterior permite tener elementos concretos para abordar el desafío de la formación de profesionales con una fuerte estructura que les permita responder a las necesidades de la sociedad en permanente cambio, pero con claras intencionalidades formativas para aportar a la experiencia del sujeto en formación y a la sociedad.

\section{Conclusiones}

Para finalizar, es importante insistir sobre la importancia de la pedagogía liderada por los docentes como sujetos protagonistas de su acción socia, desde referentes epistemológicos que se construyan y reconstruyan permanentemente a partir de las prácticas cotidianas.

También es necesario resaltar que la concepción pedagógica asume la didáctica de las disciplinas y campos desde una perspectiva investigativa para innovar el desarrollo de las metodologías y los medios, donde el docente universitario implementa una práctica docente de reflexión en la acción en la cual sistematiza sus experiencias a partir de ejes en torno el aprender y la construcción de conocimiento en la acción.

En el mismo sentido es importante retomar que la formación de profesionales reflexivos y prácticos, con formaciones pertinentes, requiere el compromiso institucional con currículos que investiguen y se alimenten con los contextos de trabajo desde una epistemología de la práctica, que incluya como componente central la reflexión en la acción y con una estructura epistemológica y disciplinar sólida. 
El currículo que se requiere para la formación en la acción implica una fundamentación disciplinar y con espacios para la formación integral, con una estructura coherente que suscite la pertinencia con el entorno nacional e internacional, que garantice la flexibilidad curricular para lograr la interdisciplinariedad en la formación y la universalidad con eficacia y equidad para lograr la inclusión de todos los sujetos y poblaciones que participen en los procesos formativos.

\section{Referencias bibliográficas}

Ainscow, M. 2005. Desarrollo de sistemas educativos inclusivos. Ponencia presentada en el Congreso Guztientzako Eskola. Gobierno Vasco, Vitoria, España.

Acevedo, S. 2013. Reflexiones sobre inclusión y educación superior. Revista de investigaciones UNAD, 12, (2): 57-67.

Acevedo, S. 2015. Perspectivas necesarias sobre educación superior inclusiva con tecnologías de la comunicación en la formación de jóvenes. En A. Álvaro y R. Rubio (coords.), Generación 2020. Madrid: Instituto de la Juventud.

Alles, M. 2012. Social Media y Recursos Humanos. Buenos Aires: Ediciones Granica.

Barbero, J. 2002. La crisis de las profesiones en la sociedad del conocimiento. En Revista Nómadas. No, 16.

Bernstein, B. 1975. Langage et classes sociales. Paris: Editions de Minuit.

Bernstein, B. 1975. Some sociological determinants of perception. British Journal of Sociology, 9: 159-174.

Bourdieu, P. 1984. Sociología y Cultura. Editorial Grijalbo: México.

Díaz, M. 2000. Pedagogía Discurso y Poder. Citado en Formación de Profesores de la Educación Superior: Programa Nacional de Oscar Ibarra, y otros. Editado por el ICFES en el 2000.

Forero, F. 1999. Mejorar la Docencia Universitaria. Tomo II. Bogotá: Editorial Universidad Pedagógica Nacional.

Fontana, J. 1992. La historia después del fin de la historia. Barcelona: Crítica.

Fuentes, C. 1998. Educación. la agenda para el Siglo XXI. Programa de las Naciones Unidas para el Desarrollo, Tercer Mundo Editores. 
Flórez, R. 1997. Hacia una pedagogía del conocimiento. Bogotá: McGraw-Hill.

Gardner, H. 2000. La educación de la mente y el conocimiento de las disciplinas. Barcelona: Editorial Paidós.

Goodman, N. 1968. Languages of art. Indianapolis: Hackett.

Habermas, J. 1982. Conocimiento e interés. Madrid: Taurus Ediciones.

Luhman, N. 1984. Sistemas Sociales. Barcelona: Anthropos- U. Iberoamericana- Centro Editorial Javeriano.

Schön, D. 1992. La formación de profesionales reflexivos: Hacia un nuevo diseño de la enseñanza y el aprendizaje de los profesionales. Temas de educación. Barcelona: Paidós.

Schütz, A. 1984. On the Origin of 'Phenomenological' Sociology, Human Studies, Vo1. (7): 163-189.

Stenhouse, L. 1984. Investigación y Desarrollo del Currículo. Madrid: Editorial. Morata.

Wulf, C. 1991. Educación II. Aportes de la Pedagogía Alemana Moderna. Quito: Proyecto EBI. 